\title{
Radio occultation experiment of the Venus atmosphere and ionosphere with the Venus orbiter Akatsuki
}

\author{
Takeshi Imamura ${ }^{1}$, Tomoaki Toda ${ }^{1}$, Atsushi Tomiki ${ }^{1}$, Daichi Hirahara ${ }^{1}$, Tomoko Hayashiyama ${ }^{1}$, Nanako Mochizuki ${ }^{1}$, \\ Zen-ichi Yamamoto ${ }^{1}$, Takumi Abe ${ }^{1}$, Takahiro Iwata ${ }^{1}$, Hirotomo Noda $^{2}$, Yoshifumi Futaana ${ }^{3}$, \\ Hiroki Ando ${ }^{4}$, Bernd Häusler ${ }^{5}$, Martin Pätzold ${ }^{6}$, and Alexander Nabatov ${ }^{7}$ \\ ${ }^{1}$ Institute of Space and Astronautical Science, Japan Aerospace Exploration Agency, 3-1-1, Yoshinodai, Sagamihara 252-5210, Japan \\ ${ }^{2}$ National Astronomical Observatory of Japan, 2-21-1 Osawa, Mitaka, Tokyo 181-8588, Japan \\ ${ }^{3}$ Swedish Institute of Space Physics, Box 812, SE-981 28, Kiruna, Sweden \\ ${ }^{4}$ The University of Tokyo, 7-3-1 Hongo, Bunkyo-ku, Tokyo 113-0033, Japan \\ ${ }^{5}$ Institut für Raumfahrttechnik, Universität der Bundeswehr München, D-85577 Neubiberg, Germany \\ ${ }^{6}$ Institut für Geophysik und Meteorologie, Universität zu Köln, D-50923, Köln, Germany \\ ${ }^{7}$ Ukrainian Academy of Science, 4, Chervonoprapornaya Str., Kharkov, 61002, Ukraine
}

(Received June 21, 2010; Revised January 26, 2011; Accepted March 22, 2011; Online published September 7, 2011)

\begin{abstract}
The Radio Science experiment (RS) in the Akatsuki mission of JAXA aims to determine the vertical structure of the Venus atmosphere, thereby complementing the imaging observations by onboard instruments. The physical quantities to be retrieved are the vertical distributions of the atmospheric temperature, the electron density, the $\mathrm{H}_{2} \mathrm{SO}_{4}$ vapor density, and small-scale density fluctuations. The uniqueness of Akatsuki RS as compared to the previous radio occultation experiments at Venus is that low latitudes can be probed many times thanks to the nearequatorial orbit. Systematic sampling in the equatorial region provides an opportunity to observe the propagation of planetary-scale waves that might contribute to the maintenance of the super-rotation via eddy momentum transport. Covering the subsolar region is essential to the understanding of cloud dynamics. Frequent sampling in the subsolar electron density also helps the understanding of ionosphere dynamics. Another unique feature of Akatsuki RS is quasi-simultaneous observations with multi-band cameras dedicated to meteorological study; the locations probed by RS are observed by the cameras a short time before or after the occultations. An ultra-stable oscillator provides a stable reference frequency which is needed to generate the X-band downlink signal used for RS.
\end{abstract}

Key words: Venus, radio science, radio occultation, exploration.

\section{Introduction}

Radio occultation is one of the major applications of radio science in space missions, and has played a central role in determining the vertical structures of planetary atmospheres from the early stage of the planetary exploration in the 1960's (e.g., Eshleman et al., 1987; Tyler, 1987). In a radio occultation experiment conducted with an orbiter, the spacecraft transmits radio waves toward a tracking station on the earth and sequentially goes behind the planet's ionosphere, neutral atmosphere, and solid planet as seen from the tracking station, and reemerges in the reverse sequence. During such occultation events the neutral and ionized atmospheres of the planet cause bending, attenuation and scintillation of the radio waves, from which information on the atmosphere along the ray path is obtained. Under the assumption of local spherical symmetry, the measurements yield vertical profiles of refractive index and absorption coefficient. The refractive index profiles yield temperature profiles by assuming hydrostatic balance. Among the

Copyright (C) The Society of Geomagnetism and Earth, Planetary and Space Sciences (SGEPSS); The Seismological Society of Japan; The Volcanological Society of Japan; The Geodetic Society of Japan; The Japanese Society for Planetary Sciences; TERRAPUB.

doi:10.5047/eps.2011.03.009 merits of this technique are its high vertical resolution and temperature resolution, which are discussed in Sections 4.4 and 5.4 .

The Radio Science experiment (RS) in the Akatsuki or Venus Climate Orbiter mission (PLANET-C project) of Japan Aerospace Exploration Agency (JAXA) will apply this technique to study the atmosphere of Venus (Nakamura et al., 2007, 2011). Although radio occultation experiments have been conducted many times in the previous Venus missions, dense sampling at low latitudes and coordination with other measurements will enable a unique, efficient observation of the atmospheric structure and its temporal variation. Radio science experiments rely on the extreme frequency stability of both the onboard radio wave source and the recording system at the ground station. Akatsuki RS employs an ultra-stable oscillator (USO) as an onboard reference frequency source.

Akatsuki, the first Venus probe of Japan, was launched on May 20 (UT), 2010, and the Venus orbit insertion maneuver for Akatsuki on December 7, 2010 has failed. At present the spacecraft is orbiting the Sun, and it will have a chance to encounter Venus 5-6 years later. JAXA is examining the possibility of conducting an orbit insertion maneuver again at this opportunity. This paper describes the onboard and 
ground-based systems, as well as the measurement principle, numerical analysis technique, and observation strategy in the original plan. These will be largely unchanged in the renewed plan.

\section{Scientific Background}

Previous radio occultation observations of the Venusian atmosphere, including those conducted by Mariner 5, Mariner 10, Pioneer Venus Orbiter (PVO) and Venus Express have provided a variety of information on the atmospheric structure. Temperature soundings by radio occultation showed that both the latitude dependence and localtime dependence are small below the cloud layer which is located around 45-70 km altitude (Kliore and Patel, 1980, 1982). Within the cloud layer $(60-70 \mathrm{~km})$ the temperature drops by $\sim 20 \mathrm{~K}$ around $60-75^{\circ}$ latitude, suggesting via thermal wind balance a prominent easterly vertical shear associated with the mid-latitude jet around $60^{\circ}$ latitude (Newman et al., 1984; Tellmann et al., 2009). This midlatitude temperature minimum surrounds the warm pole, creating the so-called polar collar (Taylor et al., 1980). The occurrence of the mid-latitude jets is consistent with the results of cloud tracking (e.g., Rossow et al., 1990). Above the cloud the temperature increases monotonically with latitude, leading to the weakening of the mid-latitude jets with height (Newman et al., 1984). The static stability is near neutral in the middle and lower cloud region (50-55 km), implying vertical convection driven by upwelling thermal radiation, while it is weakly stable below this height and strongly stable above (Fjeldbo et al., 1971; Howard et al., 1974; Tellmann et al., 2009). The overall stable stratification sustains vertical propagation of gravity waves; wavy temperature structures which might be associated with gravity waves have been observed by radio occultation (Howard et al., 1974; Hinson and Jenkins, 1995).

The mixing ratio of $\mathrm{H}_{2} \mathrm{SO}_{4}$ vapor around $35-50 \mathrm{~km}$ altitude can be measured by radio occultation by utilizing the fact that attenuation of radio waves occurs in the Venus atmosphere primarily due to $\mathrm{H}_{2} \mathrm{SO}_{4}$ vapor in this altitude region, with smaller contributions from $\mathrm{CO}_{2}$ and $\mathrm{SO}_{2}$ (Jenkins and Steffes, 1991; Jenkins et al., 1994). $\mathrm{H}_{2} \mathrm{SO}_{4}$ vapor is a key species in the global-scale sulfur cycle and the physics and chemistry of $\mathrm{H}_{2} \mathrm{SO}_{4}-\mathrm{H}_{2} \mathrm{O}$ clouds (Imamura and Hashimoto, 1998, 2001). The $\mathrm{H}_{2} \mathrm{SO}_{4}$ vapor density reaches the maximum value of $18-24 \mathrm{ppm}$ around $38-45 \mathrm{~km}$ altitude. Above this altitude the density is limited by the saturation pressure, while below this altitude it drops precipitously probably due to thermal decomposition.

Scintillations of radio waves are caused by the movement of the ray path across small-scale density structures in the atmosphere. Woo et al . (1980) analyzed the time series of the received signal power and compared their spectra with theoretical scintillation spectra based on the wave propagation theory. They found that a turbulent layer exists around $60 \mathrm{~km}$ altitude, and the scintillation power is higher at higher latitudes. An eddy diffusion coefficient of $4 \mathrm{~m}^{2} \mathrm{~s}^{-1}$ was estimated for $60 \mathrm{~km}$ altitude from the same data set. Leroy and Ingersoll (1996) attributed the scintillations to vertically-propagating gravity waves created by cloud-level convection.
The height range of the Venus' neutral atmosphere accessible by radio occultation is approximately $32-90 \mathrm{~km}$. Below $40 \mathrm{~km}$ the defocusing loss and the attenuation by the atmosphere limits the quality of the observation, and $32 \mathrm{~km}$ is the lowest accessible altitude, below which the radius of curvature of the ray path becomes smaller than the distance to the planet center (Fjeldbo et al., 1971; Häusler et al., 2006). The atmosphere above $100 \mathrm{~km}$ is too thin to be detected. The broad height coverage of radio occultation is a great advantage in the investigation of the vertical coupling among different atmospheric layers; no other technique can cover this altitude range at the same time.

At altitudes above $\sim 100 \mathrm{~km}$ the electrons in the ionosphere are the major contributors to the ray bending. The peak electron density occurs at around $150 \mathrm{~km}$ altitude, and is on the order of $10^{5} \mathrm{~cm}^{-3}$ on the dayside and $10^{4} \mathrm{~cm}^{-3}$ on the nightside (Kliore, 1992; Pätzold et al., 2007a). The Venusian nightside ionosphere is thought to be generated by the transportation of electrons from the dayside and the high energy electron impact on the nightside. The ionosphere of Venus is not protected by the planet's intrinsic magnetic field from the impinging solar wind, and thus the thermal and magnetic pressure of the ionosphere will balance the dynamic pressure of the solar wind at the upper boundary of the ionosphere called the ionopause. Previous observations revealed that the ionopause is located at $200-400 \mathrm{~km}$, except for the high solar zenith angle (SZA) ionosphere with SZA $>50^{\circ}$ during the solar maximum, when the ionopause is located at $200-1000 \mathrm{~km}$ and the height is highly variable. Frequent measurements at low SZA will complement the previous observations, which were conducted mostly at SZA $>30^{\circ}$ (Kliore and Luhmann, 1991). The structure of the ionosphere is affected by the input of energy and momentum from the solar wind to the upper atmosphere, and thus important for understanding the escape of the atmosphere to space, which should have driven the climate evolution of Venus in a geological time scale.

\section{Objectives}

The primary goal of Akatsuki RS is to characterize the meteorological processes that might drive the planet-wide easterly wind (super-rotation) as well as to understand the cloud dynamics. For this purpose the temporal and spatial variability of the Venus atmosphere will be studied by retrieving the vertical profiles of the temperature, the $\mathrm{H}_{2} \mathrm{SO}_{4}$ vapor density, and the intensity of small-scale density fluctuations.

The advantage over the previous missions is the nearequatorial orbit of the spacecraft and a suite of multiband cameras dedicated to meteorological study (Nakamura et al., 2007, 2011). The planned orbit around Venus is a 30 hour-period elliptical orbit near the ecliptic plane with the periapsis latitude of $\sim 10.5^{\circ} \mathrm{S}$ and the apoapsis latitude of $\sim 10.5^{\circ} \mathrm{N}$. The direction of orbital motion is westward, which coincides with the direction of the atmospheric super-rotation. The apoapsis altitude is chosen to be $78500 \mathrm{~km}$, or 13 Venus radii $\left(R_{\mathrm{V}}\right)$, so that the angular velocity of the spacecraft is roughly synchronized with the $60-\mathrm{m} \mathrm{s}^{-1}$ super-rotational flow near the cloud base $(50 \mathrm{~km})$ for $\sim 20$ hours centered at the apoapsis. The periapsis alti- 

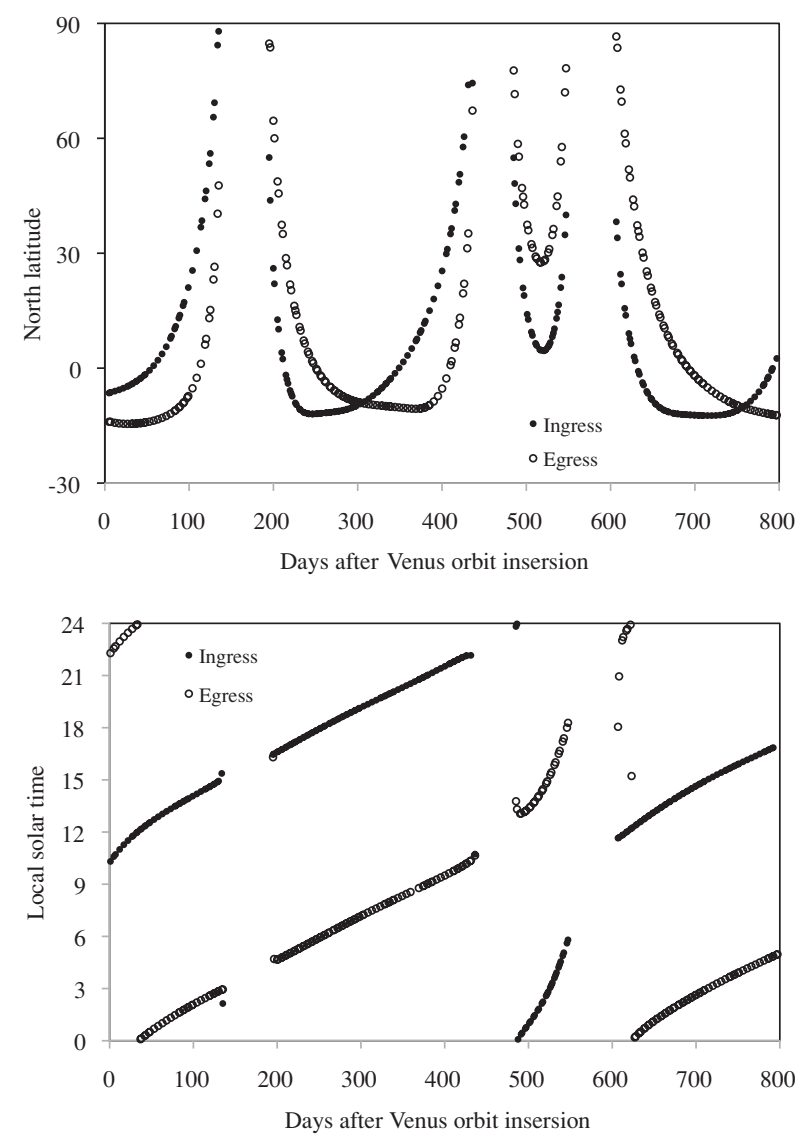

Fig. 1. (upper) Latitudes and (lower) local solar times of the observation points during the nominal mission period in the original plan. Filled circles and open circles indicate ingress and egress occultations, respectively. The observation point is defined as the tangential point of the straight line between the spacecraft and the ground antenna at the moment when the spacecraft is occulted by the solid surface of Venus.

tude is $\sim 600 \mathrm{~km}$.

Figure 1 shows the latitude and local time of the observation in the original plan as a function of the Earth days after Venus orbit insertion (VOI), which was scheduled for early December 2010, during the nominal mission period of $\sim 2$ Earth years. Approximately 200 experiments, each of which includes an ingress and an egress occultations, are planned to be conducted during this 2 years. The uniqueness of the Akatsuki RS is that probed points cluster around the equatorial region. Since the onboard cameras are designed to observe wide areas centered at the equatorial region, the locations probed by $\mathrm{RS}$ can be observed by the cameras a short time (minutes to hours) before or after the occultations. The vertical profiles taken by RS and the horizontally-resolved information at several height levels by the cameras complement each other in developing three-dimensional models of the atmosphere.

Systematic long-term sampling on a specific latitudinal band (equatorial region) also provides a unique opportunity to study the propagation of planetary-scale waves such as Kelvin waves and thermal tides, which might play essential roles in sustaining the super-rotation (e.g., Rossow et al., 1990; Yamamoto and Tanaka, 1997; Takagi and Matsuda, 2007). Typically an occultation (an ingress-egress pair) is observed every 3-4 days from the tracking station. Given this sampling interval, planetary-scale waves with 46 days period, which have been detected at the cloud top (e.g., Rossow et al., 1990) would be observed apparently as much longer period (up to several tens of days) waves due to aliasing. However, such long periods correspond to very fast intrinsic phase speeds because the waves observed from the inertial frame are Doppler-shifted by the 4 dayperiod super-rotation. Such a solution will be rejected based on meteorological considerations, and more realistic waves with periods of several days will be obtained by fitting a sinusoidal function to the data.

The almost complete local-time coverage in the low latitude (Fig. 1) enables the studies of equatorial cloud dynamics which is poorly understood. For example, cell-like structures have been observed near the subsolar region in ultraviolet (Rossow et al., 1980; Markiewicz et al., 2007). The origin of these structures has been unclear, because their horizontal scales of up to several hundreds kilometers seem to be too large for convective cells and the cloud top region is considered to be basically stably stratified (Baker and Schubert, 1992; Toigo et al., 1994). The diurnal variation of the temperature profile to be revealed by RS, especially the possible variation in the depth of the neutralstability layer, should become a key to understanding the cloud dynamics in this region.

Excursions of the probed points to the northern high latitude occur several times and enable observations of the latitudinal structure. Since the Venus atmosphere is considered to be in cyclostrophic balance, the meridional distribution of the zonal wind can be derived from the temperature distribution by integrating the thermal wind equation with an appropriate lower boundary condition (Newman et al., 1984). This zonal wind distribution will become the basis for understanding the momentum balance of the atmospheric general circulation. The combination of data from Akatsuki RS with those from ESA's Venus Express radio science VeRa (Häusler et al., 2006; Pätzold et al., 2007a; Tellmann et al., 2009), which conducts dense sampling in the high latitude by virtue of the polar orbit, would also enable studies of meteorological processes over broad latitude regions even when the two spacecraft do not orbit at the same time.

Another goal of Akatsuki RS is to study the spatial and temporal variabilities of the ionosphere in response to the variations in the solar wind and the neutral atmosphere. Although Akatsuki is not equipped with a magnetometer and particle instruments, Venus Express has those instruments and would provide supplementary information (Zhang et al., 2006; Barabash et al., 2007) if Venus Express mission is extended till Akatsuki's next Venus orbit insertion. Such a configuration is very optimal for studying the response of the ionosphere to the solar wind condition, because the upstream solar wind conditions and their sudden changes such as interplanetary shock passages and coronal mass ejections can be monitored simultaneously. Sudden changes in the solar wind modify the global characteristics of the Venusian ionosphere, which may increase the ion escape flux from the upper atmosphere (Futaana et al., 2008; Edberg et al., 2010). Energetic electrons at energies $\approx 30 \mathrm{eV}$ (Kliore et 


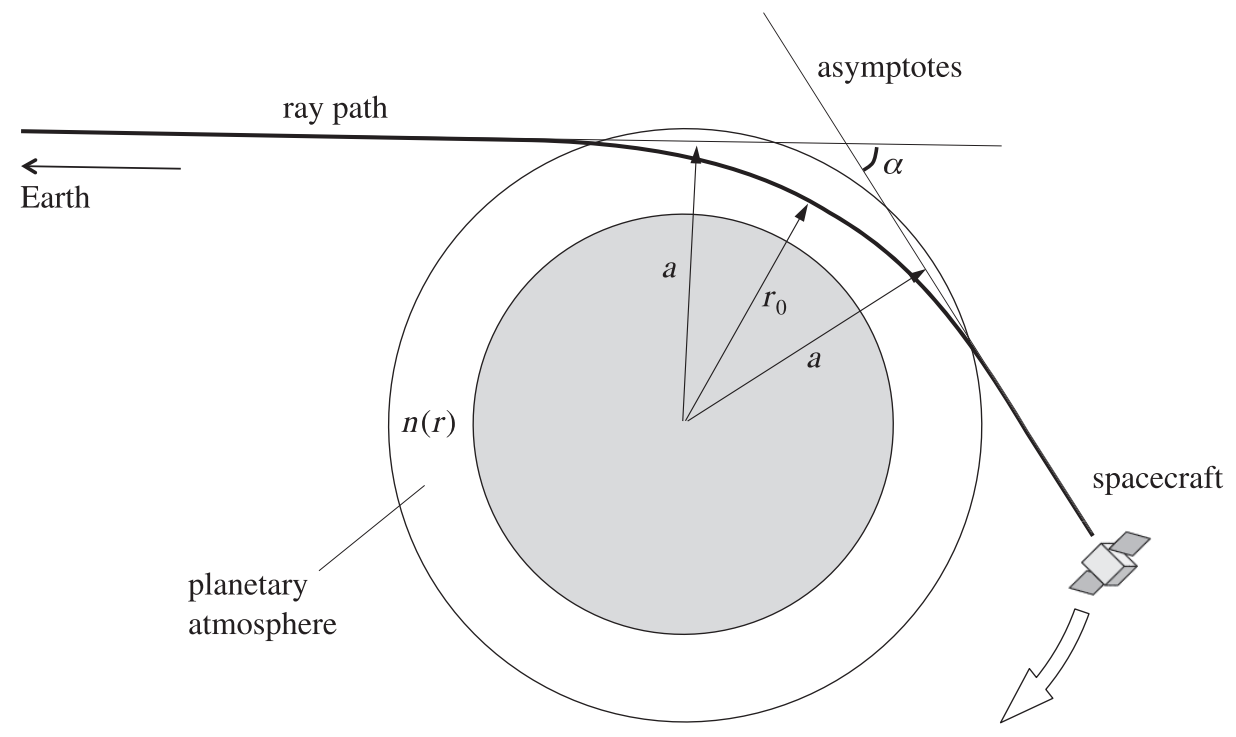

Fig. 2. Ray bending in a planetary atmosphere (see text).

$a l ., 1991)$, considered as one of the generation mechanisms of the nightside ionosphere, may also be observed by an electron spectrometer onboard Venus Express.

Extensive measurements of the ionosphere at small SZA allow us to understand how the upper atmosphere interacts with the solar wind in the subsolar region. The investigation of the subsolar region is important for understanding the transfer of energy and momentum from the solar wind to the upper atmosphere. In situ observations of the subsolar region have been difficult, because spacecraft normally pass through very quickly at ionospheric altitudes.

Studying the properties of the solar wind plasma is also an objective of Akatsuki RS. When the spacecraft moves into superior conjunction with the Sun, radio waves transmitted from the spacecraft toward the tracking station suffer bending, scattering and attenuation in the near-sun plasma. The temporal variations of the phase and the intensity of the received signal provide information on the spatial spectrum (power law) of density inhomogeneities, the anisotropy of inhomogeneities, the inner turbulence scale, and the bulk velocity of plasma (Pätzold et al., 1996; Imamura et al., 2005; Efimov et al., 2010). Based on the noise level estimation (Section 5.4) and previous occultation results (Woo and Armstrong, 1979), we expect the phase power spectrum will be obtained up to $\sim 10 \mathrm{~Hz}$ for the solar offset distance of 10 solar radii; such a spectrum would enable us to study, for example, the excess power at small scales $(100-600 \mathrm{~km})$ observed by the Nozomi radio occultation (Imamura et al., 2005). The minimum solar offset distance of $\sim 1.5$ solar radii will occur on June 25, 2011 in the renewed cruising plan.

\section{Observation Principles}

\subsection{Radio wave propagation in the atmosphere}

The radio wave transmission mode for Akatsuki RS, shown in Fig. 2, is a one-way downlink at X-band
$(8.410926 \mathrm{GHz})$. It is known that for a radially symmetric atmosphere the asymptotic bending angle $\alpha$ is related to the refractive index $n$ as (e.g., Fjeldbo et al., 1971)

$$
\alpha(a)=-2 a \int_{r_{0}}^{\infty} \frac{1}{n} \frac{\partial n}{\partial r} \frac{d r}{\sqrt{(n r)^{2}-a^{2}}},
$$

where $a$ is the ray impact parameter, $r$ is the radius, and $r_{0}$ is the radius of the closest approach given by the Bouguer's rule

$$
n\left(r_{0}\right) r_{0}=a \text {. }
$$

Using (1), it can be shown that $n$ is obtained as a function of $r$ from the relationship between $\alpha$ and $a$ through an Abel transformation

$$
\ln n(r)=-\frac{1}{\pi} \int_{a_{1}}^{\infty} \ln \left\{\frac{a}{a_{1}}+\left[\left(\frac{a}{a_{1}}\right)^{2}-1\right]^{\frac{1}{2}}\right\} \frac{d \alpha}{d a} d a,
$$

where $a_{1}$ represents the impact parameter for a ray whose radius of closest approach is $r$. Both $\alpha$ and $a$ can be calculated from the measured atmospheric Doppler shift and the reconstructed velocity and position vectors of the spacecraft and the ground antenna.

Figure 3 shows the relationship between the bending angle $\alpha$ and the impact parameter $a$ as well as the relationship between $\alpha$ and the ray closest distance $r_{0}$ calculated with (1) for the Venus International Reference Atmosphere (Seiff et al., 1985) combined with a Gaussian-type model ionosphere with the peak electron density of $5 \times 10^{5} \mathrm{~cm}^{-3}$, the peak height of $150 \mathrm{~km}$ and the width of $30 \mathrm{~km}$. The minimum $a$ corresponds to a height above the surface of $\sim 45 \mathrm{~km}$, which gives the altitude of the ray closest approach of $\sim 32 \mathrm{~km}$ when the radius of the planet is taken to be $6052 \mathrm{~km}$. At this height the atmosphere is critically refractive, making the lower atmosphere inaccessible to radio occultation. 

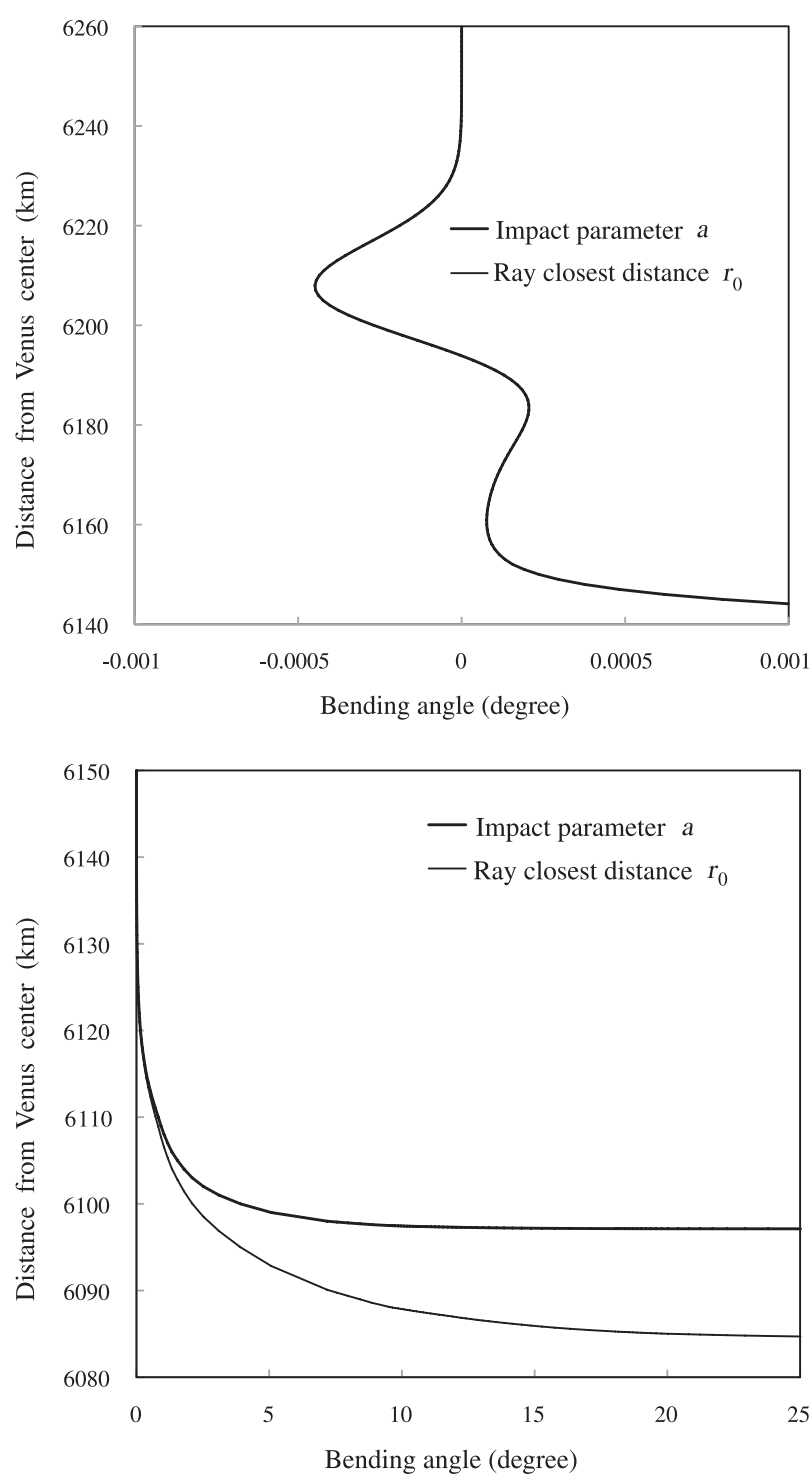

Fig. 3. Relationship between the bending angle $\alpha$ and the impact parameter $a$ (thick curve) and that between the bending angle and the ray closest distance $r_{0}$ (thin curve) for a model ionosphere described in the text and the "VIRA" neutral atmosphere model. The radius of Venus is taken to be $6052 \mathrm{~km}$. (upper) Plot in the ionospheric altitude range, where the thick and thin curves almost overlap. (lower) Plot in the neutral atmosphere range.

\subsection{Derivation of atmospheric density and tempera-} ture

The refractivity $\mu$ is related to the refractive index $n$ by

$$
\mu=(n-1) \times 10^{6} .
$$

The $\mu$ is the sum of the contributions from the neutral atmosphere and the ionosphere:

$$
\mu=V N-\frac{\beta N_{\mathrm{e}}}{f^{2}} \times 10^{6},
$$

where $V$ is the refractive volume, $N$ is the number density of the neutral atmosphere, $\beta=e^{2} / 8 \pi^{2} \epsilon m_{\mathrm{e}} \sim 40.3$ $\mathrm{m}^{3} \mathrm{~s}^{-2}$ with $e, \epsilon$ and $m_{\mathrm{e}}$ being the elementary charge, dielectric constant in vacuum and electron mass, respectively, $c$ is the speed of light, $N_{\mathrm{e}}$ is the number density of electrons, and $f$ is the frequency of the carrier signal. On the assumption that the neutral atmosphere is well mixed, we adopt a constant $V$ of $1.81 \times 10^{-17} \mathrm{~m}^{3}$ based on the composition of $96.5 \% \mathrm{CO}_{2}$ and $3.5 \% \mathrm{~N}_{2}$ using the constants given in Fjeldbo and Eshleman (1968). The neutral and ionospheric contributions are separated in altitude with the boundary around $100 \mathrm{~km}$ altitude (see results of Pätzold et al., 2007a). This enables us to retrieve the vertical profiles of the neutral atmospheric density $N$ and the electron density $N_{\mathrm{e}}$ separately.

The vertical profile of the neutral atmospheric pressure, $p(r)$, is derived from the density profile $N(r)$ by integrating the equation of hydrostatic equilibrium:

$$
p(r)=p\left(r_{\text {top }}\right)+m \int_{r}^{r_{\text {top }}} N\left(r^{\prime}\right) g\left(r^{\prime}\right) d r^{\prime},
$$

where $m$ is the mean molecular mass, $g$ is the acceleration due to gravity, and $r_{\text {top }}$ is the adopted upper boundary. The ideal gas law relates $p\left(r_{\text {top }}\right)$ to the atmospheric temperature $T$ at this height as

$$
p\left(r_{\text {top }}\right)=N\left(r_{\text {top }}\right) k T\left(r_{\text {top }}\right),
$$

where $k$ is the Boltzmann's constant. The boundary condition required for the integration is the value $T\left(r_{\text {top }}\right)$. The temperature profile $T(r)$ is calculated from $N(r)$ and $p(r)$ using the ideal gas law. The influence of the adopted $T\left(r_{\text {top }}\right)$ on the calculated $T(r)$ is almost negligible except at the uppermost $10 \mathrm{~km}$ (Pätzold et al., 2007a; Tellmann et al., 2009).

\subsection{Atmospheric defocusing and absorption}

The received signal power varies during each occultation experiment due to atmospheric defocusing and absorption. The former must be taken into account when retrieving the vertical distribution of absorbing materials from the observed signal power.

Atmospheric defocusing is caused by the radial gradient of the refractive index. The defocusing loss is estimated by (Eshleman, 1973)

$$
L=\left(\cos \alpha-D \frac{d \alpha}{d a}\right)^{-1}
$$

where $D$ is the distance from the spacecraft to the crossing of the ray asymptotes. Figure 4 shows the expected defocusing loss in units of $\mathrm{dB},-10 \log L$, as a function of the ray closest distance $r_{0}$ for $D=1,2$, and $5 R_{\mathrm{V}}$. The loss increases with $D$, and it is as large as $\sim 26 \mathrm{~dB}$ at $40 \mathrm{~km}$ altitude $(6092 \mathrm{~km}$ from the planet center) for a typical $D$ of $2 R_{\mathrm{V}}$.

Once the defocusing loss is estimated as a function of time from trajectory information and the relationship between $\alpha$ and $a$ from the Doppler measurements, one may calculate the total atmospheric absorption from the time series of the received signal power. This time series is converted to a height profile of the absorptivity with the aid of ray tracing on the assumption of a spherically symmetric atmosphere (Jenkins and Steffes, 1991; Jenkins et al., 1994). The absorption due to $\mathrm{CO}_{2}, \mathrm{~N}_{2}$ and $\mathrm{SO}_{2}$ is removed from the absorptivity profile based on its dependence on the ambient pressure and temperature. The remaining absorption will be principally due to $\mathrm{H}_{2} \mathrm{SO}_{4}$ vapor. 


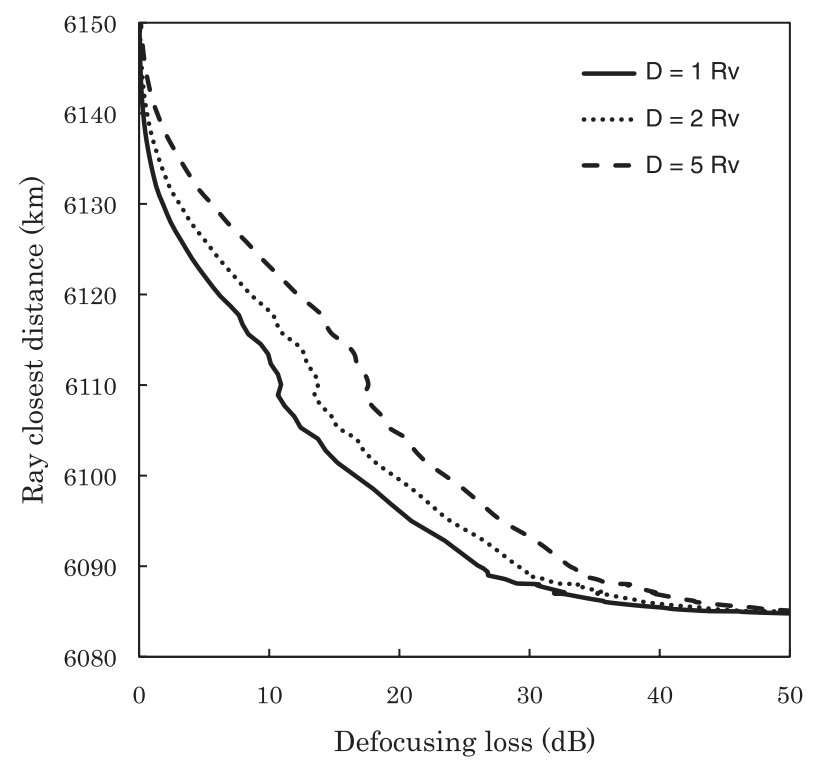

Fig. 4. Defocusing loss as a function of the ray closest distance $r_{0}$ for three distances from the spacecraft to the crossing of the ray asymptotes, $D$.

\subsection{Vertical and horizontal resolution}

The vertical resolution is limited by the diameter of the first Fresnel zone. The refraction in the atmosphere changes the Fresnel zone shape from circular to elliptical form, and the resulting vertical diameter is (Fjeldbo and Eshleman, 1969; Karayel and Hinson, 1997)

$$
d_{\mathrm{v}}=2 \sqrt{\lambda D L},
$$

where $\lambda \sim 3.6 \mathrm{~cm}$ is the wavelength of the carrier signal. Here $D$ is assumed to be much smaller than the distance from the planet center to the receiver. For a typical $D$ of $2 R_{\mathrm{V}}$ and without defocusing loss $(L=1)$, we obtain $d_{\mathrm{v}} \sim$ $1.3 \mathrm{~km}$. When defocusing loss is $\sim 26 \mathrm{~dB}$ at $40 \mathrm{~km}$ altitudes, we obtain $d_{\mathrm{v}} \sim 60 \mathrm{~m}$ for the same $D$.

Letting the typical scale height of $H=6 \mathrm{~km}$ (corresponding to $60 \mathrm{~km}$ altitude) and the planetary radius of $R=6052 \mathrm{~km}$, and ignoring the bending of the ray path, the length of the ray path traversing the atmospheric layer with a thickness $H$ is $2\left((R+H)^{2}-R^{2}\right)^{1 / 2} \sim 500 \mathrm{~km}$. This length is considered the horizontal resolution of the measurement because the atmospheric variations are smeared out approximately within this interval. The orientation of the radio path is near perpendicular to the meridian in most of the cases and the smearing of atmospheric variations occurs along this direction.

\section{Observation System}

\subsection{Radio communication system}

Figure 5 shows a block diagram of the Akatsuki onboard radio communication system. The downlink frequency is $8.410926 \mathrm{GHz}$ (X-band) only. During the experiments, one of the X-band transponders (XTRP) is locked to an ultra-stable oscillator (USO), and the transponder signal is amplified by the $23.4 \mathrm{~W}$ traveling wave tube amplifier (TWTA). The $8.7 \mathrm{~W}$ solid state power amplifiers (SSPA) will not be used in the experiments unless we have trouble with the TWTA. The gain of the 0.9 m-diameter flat-type slot array antenna (XHGA-T) is $35 \mathrm{dBi}$, corresponding to a $3 \mathrm{~dB}$ full beam width of $\sim 2^{\circ}$. Since the ray bending far exceeds the beam width (Fig. 2), the spacecraft will perform attitude maneuvers to compensate for the changing direction of the signal path. The equivalent isotropically radiated power (EIRP) is $42.5 \mathrm{dBW}$ when the TWTA is used.

The radio waves transmitted by the spacecraft will be received at the Usuda Deep Space Center (UDSC), which is located at $138^{\circ} 21^{\prime} 54^{\prime \prime}$ East longitude, $36^{\circ} 07^{\prime} 44^{\prime \prime}$ North latitude in Japan. The $64-\mathrm{m}$ dish provides a gain of $72.5 \mathrm{dBi}$ including the feeder loss in X-band, at a system noise temperature of $96 \mathrm{~K}$ for a $5 \mathrm{~mm}_{\text {hour }}^{-1}$ rain and an elevation angle of $15^{\circ}$. The recording system to be used is the 'IPVLBI recorder' which was developed for radio astronomy. The received signals are down-converted to several hundred $\mathrm{kHz}$ by an open-loop heterodyne system stabilized by a hydrogen maser (Allan deviation $<3 \times 10^{-13}$ for $1 \mathrm{~s},<3 \times 10^{-15}$ for $1000 \mathrm{~s}$ ) and 8-bits digitized. The sampling rate will be $1 \mathrm{MHz}$ or more so that the carrier signal, whose frequency changes by $\sim 100 \mathrm{kHz}$ or more during each occultation experiment, can be confined in the recording bandwidth.

\subsection{Ultra-stable oscillator (USO)}

The USO (Fig. 6) provides a stable reference frequency (38.22567759 MHz) for the transponder. It was manufactured by TimeTech GmbH in Germany and is a heritage from the USOs flown onboard the ESA's Rosetta (Pätzold et al., 2007b) and Venus Express spacecraft (Häusler et al., 2006). We made several modifications such as the redesign of the thermal insulating and mechanical shock protection system to adapt to the higher mechanical loads during the launch, resulting in dimensions $(17 \mathrm{~cm} \times 13 \mathrm{~cm} \times 16 \mathrm{~cm})$ and mass $(1.9 \mathrm{~kg})$ which are greater than for previous USOs. The USO is characterized by an excellent frequency stability; the Allan deviation is on the order of $10^{-13}$ at the integration time from 1 to $1000 \mathrm{~s}$.

\subsection{Signal processing}

The phase fluctuation will be retrieved from the recorded signal in the following manner (Imamura et al., 2005). In order to suppress the frequency variation to enable narrowband filtering, a predicted Doppler frequency based on a model atmosphere is subtracted from the original signal by heterodyning. Then, approximate carrier frequencies are determined for successive time blocks by fitting theoretical signal spectra to the discrete Fourier transforms of the voltage variation in successive blocks (Lipa and Tyler, 1979). This frequency time series is divided into another set of successive blocks with time intervals of a few seconds, and smooth functions (such as spline) are determined to approximate the frequency variations in these blocks. Then the resultant frequency variation corresponding to the smooth functions is subtracted from the signal by heterodyning to apply another narrow-band filter.

The filtering scheme automatically converts the data to a complex representation, in which each datum comprises both the amplitude and phase of the signal. The low-noise signal generated by narrow-band filterings yields the phase by removing cycle slips via the phase unwrapping procedure (Mizuno et al., 1992). The frequency is obtained by 


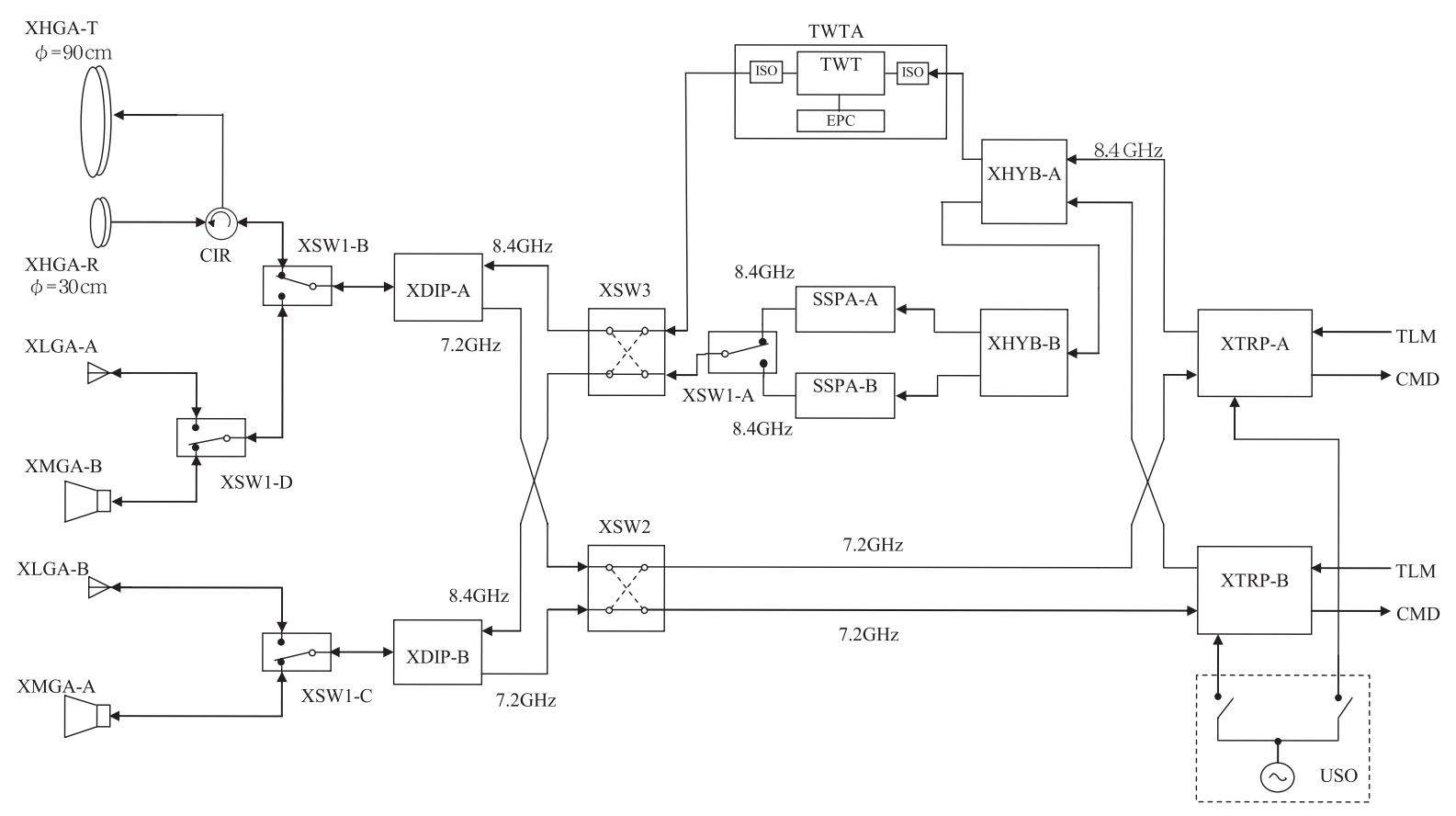

Fig. 5. Block Diagram of the Akatsuki communication subsystem (Courtesy of NEC TOSHIBA Space Systems, Ltd.).

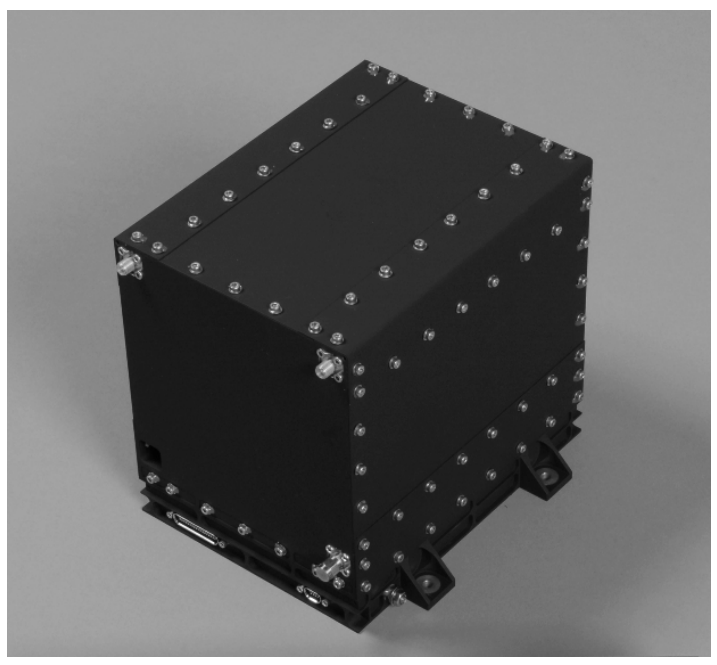

Fig. 6. Photograph of the USO onboard Akatsuki.

differentiating the phase with respect to time. The sum of the frequency variation in this final product and the frequency variations that have been subtracted via heterodyning in the course of the processing gives the total atmospheric Doppler shift, from which the bending angle is calculated.

\subsection{Link budget and error estimation}

The distance between the Earth and Venus at occultation opportunities ranges from $0.35 \mathrm{AU}$ (Astronomical unit) to $1.73 \mathrm{AU}$. Table 1 presents the link budgets including the defocusing and absorption losses, for the minimum and maximum Earth-Venus distances, and for the heights of the ray closest approach of 40, 60 and $80 \mathrm{~km}$. The absorption loss is taken from Häusler et al. (2009).

The carrier-to-noise density ratio $\left(C / N_{0}\right)$ of the received signal decreases significantly at low altitudes $(40 \mathrm{~km})$. This
Table 1. LInk budget of Akatsuki RS including losses for two Venus-Earth distances and for the minimum probed altitudes of 40, 60 and $80 \mathrm{~km}$.

\begin{tabular}{|c|c|c|}
\hline Distance (AU) & 0.35 & 1.73 \\
\hline EIRP of transmission (dBW) & \multicolumn{2}{|c|}{42.5} \\
\hline Absorption and rain loss (dB) & \multicolumn{2}{|c|}{-0.8} \\
\hline Receiver pointing loss $(\mathrm{dB})$ & \multicolumn{2}{|c|}{-0.2} \\
\hline$G / T$ of ground station $(\mathrm{dB} / \mathrm{K})$ & \multicolumn{2}{|c|}{52.7} \\
\hline$C / N_{0}$ without Venus atmosphere $(\mathrm{dBHz})$ & 57.5 & 43.6 \\
\hline \multicolumn{3}{|l|}{ Defocusing loss for $D=2 R \mathrm{v}(\mathrm{dB})$} \\
\hline $40 \mathrm{~km}$ & \multicolumn{2}{|c|}{-25.9} \\
\hline $60 \mathrm{~km}$ & \multicolumn{2}{|c|}{-12.6} \\
\hline $80 \mathrm{~km}$ & \multicolumn{2}{|c|}{-2.1} \\
\hline \multicolumn{3}{|l|}{ Absorption loss $(\mathrm{dB})$} \\
\hline $40 \mathrm{~km}$ & \multicolumn{2}{|c|}{-14.7} \\
\hline $60 \mathrm{~km}$ & \multicolumn{2}{|c|}{-0.5} \\
\hline $80 \mathrm{~km}$ & \multicolumn{2}{|c|}{0.0} \\
\hline \multicolumn{3}{|l|}{ Overall $C / N_{0}(\mathrm{dBHz})$} \\
\hline $40 \mathrm{~km}$ & 16.9 & 3.0 \\
\hline $60 \mathrm{~km}$ & 44.4 & 30.5 \\
\hline $80 \mathrm{~km}$ & 55.4 & 41.5 \\
\hline
\end{tabular}

low $C / N_{0}$ will be partially compensated by a long integration time, since at low altitudes the vertical movement of the ray path slows down due to the bending of the ray path. Moreover, the greater reflectivity of the lower atmosphere results in a higher sensitivity of the bending angle to the atmospheric temperature.

The statistical (random) uncertainty in the temperature should be on the order of $0.1 \mathrm{~K}$ or smaller for a prescribed vertical resolution of $1 \mathrm{~km}$ in the altitude region from $40 \mathrm{~km}$ to $80 \mathrm{~km}$ so that various atmospheric waves can be detected. To estimate the $C / N_{0}$ required for this purpose, we have computed the response of the downlink frequency to Gaussian-type temperature perturbations with a height of 
$0.1 \mathrm{~K}$ and a full-width of $1 \mathrm{~km}$ assuming an occultation geometry in which the ray transverse velocity relative to the Venus limb is its maximum, i.e. the case where the maximum time resolution is needed. The results showed that such temperature perturbations cause frequency perturbations roughly localized around the perturbed region. For altitudes around $80 \mathrm{~km}$, a frequency resolution of $\sim 0.03 \mathrm{~Hz}$ and a time resolution of $\sim 0.1 \mathrm{~s}$ are needed to discriminate perturbations imposed on adjacent two layers separated by $1 \mathrm{~km}$; this corresponds to a phase resolution of $\sigma \sim 0.019$ radians with a bandwidth of $B \sim 10 \mathrm{~Hz}$, giving a required $C / N_{0}$ of $B / 2 / \sigma^{2} \sim 41.5 \mathrm{dBHz}$. This value will be achieved according to the link budget analysis (Table 1). Similar analyses for $60 \mathrm{~km}$ and $40 \mathrm{~km}$ altitudes showed that the required $C / N_{0}$ is lower than the predicted $C / N_{0}$ for these altitudes. From these results we expect that the statistical error of the order of $0.1 \mathrm{~K}$ is possible. The influence of the fluctuation in the USO output frequency is negligible.

The systematic uncertainty in the temperature is difficult to estimate and is not presented in this paper. The sources of the error include the error in the spacecraft position, deviation from spherical symmetry of the atmosphere, longtimescale fluctuation of the transmit frequency, and possible multipath effect. An error estimation method given by Lipa and Tyler (1979) would be valid when the statistical characteristics of these errors are provided.

The accuracy of electron density measurement is limited at least by the fluctuation of the terrestrial ionosphere and is estimated to be on the order of $100 \mathrm{~cm}^{-3}$ based on the measurement of the ionospheric column density (Imamura et al., 2010). The density fluctuation of the interplanetary plasma can also be an error source for small solar offset distances of the ray path. Based on the results of solar occultation measurements (Woo and Armstrong, 1979; Imamura et $a l ., 2005)$, the error is estimated to be $\sim 3000 \mathrm{~cm}^{-3}$ for the solar offset distance of $0.1 \mathrm{AU}$, and decreases to $\sim 300 \mathrm{~cm}^{-3}$ for $0.3 \mathrm{AU}$, given the typical time scale of traversing the Venus ionosphere of $10 \mathrm{~s}$. It should be noted that these uncertainties vary with time.

\section{Summary}

A radio science investigation (RS) of the Venusian atmosphere will be conducted in the JAXA's Akatsuki mission. The observation targets of Akatsuki RS are (i) atmospheric temperature profiles for studying the thermal structure and atmospheric waves, (ii) sub-cloud $\mathrm{H}_{2} \mathrm{SO}_{4}$ vapor profiles for studying cloud physics, (iii) scintillation intensities for studying turbulence or gravity wave parameters, and (iv) ionospheric electron density profiles for studying ionosphere-solar wind interaction.

To achieve a high frequency stability in the X-band downlink signal, the spacecraft is equipped with an USO as a reference frequency source. During occultations the spacecraft will perform attitude maneuvers to compensate for the ray bending. The downlink signal will be recorded by an open loop receiver at the Usuda Deep Space Center of Japan. The frequency and intensity variations observed at the tracking station will be processed off-line, and the vertical structure of the Venus atmosphere will be derived assuming local spherical symmetry of the atmosphere.
The uniqueness of Akatsuki RS as compared to the previous radio occultation experiments at Venus is that low latitudes can be probed many times thanks to the nearequatorial orbit of the spacecraft. Systematic long-term sampling in the equatorial region provides a opportunity to observe the propagation of planetary-scale waves such as Kelvin waves and thermal tides, which might contribute to the maintenance of the super-rotation via eddy momentum transport. Covering the subsolar region is essential to the understanding of the cloud dynamics. Another advantage of Akatsuki RS is a suite of multiband cameras dedicated to meteorological study; the locations probed by RS will be observed by the cameras a short time before or after the occultations. The vertical profiles by RS and the horizontally-resolved information by the cameras complement each other in developing three-dimensional models of the atmosphere.

The Venus orbit insertion maneuver for Akatsuki on December 7, 2010 has failed. At present the spacecraft is orbiting the Sun, and it will have a chance to encounter Venus 5-6 years later. JAXA is examining the possibility of conducting an orbit insertion maneuver again at this opportunity.

Acknowledgments. We would like to acknowledge all members of the Akatsuki project team and the staff of UDSC for supporting the preparation of the experiment. The high performance of the USO was achieved by the tremendous efforts made by Theo Schwall, Wolfgang Schäfer and other engineers of TimeTech $\mathrm{GmbH}$. This experiment would not have become possible without the assistance of the engineers of NEC TOSHIBA Space Systems, Ltd. We also thank M. Bird and an anonymous reviewer for carefully reviewing the original manuscript and providing constructive suggestions.

\section{References}

Baker, R. D. and G. Schubert, Cellular convection in the atmosphere of Venus, Nature, 355, 710-712, 1992.

Barabash, S., J.-A. Sauvaud, H. Gunell, H. Andersson, A. Grigoriev, K. Brinkfeldt, M. Holmström, R. Lundin, M. Yamauchi, K. Asamura, W. Baumjohann, T. L. Zhang, A. J. Coates, D. R. Linder, D. O. Kataria, C. C. Curtis, K. C. Hsieh, B. R. Sandel, A. Fedorov, C. Mazelle, J.-J. Thocaven, M. Grande, H. E. J. Koskinen, E. Kallio, T. Säles, P. Riihela, J. Kozyra, N. Krupp, J. Woch, J. Luhmann, S. McKenna-Lawlor, S. Orsini, R. Cerulli-Irelli, M. Mura, M. Milillo, M. Maggi, E. Roelof, P. Brandt, C. T. Russell, K. Szego, J. D. Winningham, R. A. Frahm, J. Scherrer, J. R. Sharber, P. Wurz, and P. Bochsler, The analyser of space plasmas and energetic atoms (ASPERA-4) for the Venus Express mission, Planet. Space Sci., 55, 1772-1792, 2007.

Edberg, N. J. T., H. Nilsson, A. O. Williams, M. Lester, S. E. Milan, S. W. H. Cowley, M. Fränz, S. Barabash, and Y. Futaana, Pumping out the atmosphere of Mars through solar wind pressure pulses, Geophys. Res. Lett., 37, L03107, doi:10.1029/2009GL041814, 2010.

Efimov, I., T. Imamura, K.-I. Oyama, K. Noguchi, L. N. Samoznaev, A. S. Nabatov, M. K. Bird, and I. V. Chashei, Properties of the turbulence of the solar wind from radio-eclipse experiments with the NOZOMI spacecraft, Astron. Rep., 54, 1031-1041, 2010.

Eshleman, V. R., The radio occultation method for the study of planetary atmospheres, Planet. Space Sci., 21, 1521-1531, 1973.

Eshleman, V. R., D. P. Hinson, G. F. Lindal, and G. L. Tyler, Past and future of radio occultation studies of planetary atmospheres, Adv. Space Res., 7(12), 29-32, 1987.

Fjeldbo, G. and V. R. Eshleman, The atmosphere of Mars analyzed by integral inversion of the Mariner IV occultation data, Planet Space Sci., 16, 1035-1059, 1968.

Fjeldbo, G. and V. R. Eshleman, Atmosphere of Venus as studied with the Mariner 5 dual radio-frequency occultation experiment, Radio Sci., $\mathbf{4}$, 879-897, 1969.

Fjeldbo, G., A. J. Kliore, and V. R. Eshleman, The neutral atmosphere 
of venus as studied with the Mariner $\mathrm{V}$ radio occultation experiments, Astron. J., 76, 123-140, 1971.

Futaana, Y., S. Barabash, M. Yamauchi, S. McKenna-Lawlor, R. Lundin, J. G. Luhmann, D. Brain, E. Carlsson, J.-A. Sauvaud, J. D. Winningham, R. A. Frahm, P. Wurz, M. Holmström, H. Gunell, E. Kallio, W. Baumjohann, H. Lammer, J. R. Sharber, K. C. Hsieh, H. Andersson, A. Grigoriev, K. Brinkfeldt, H. Nilsson, K. Asamura, T. L. Zhang, A. J. Coates, D. R. Linder, D. O. Kataria, C. C. Curtis, B. R. Sandel, A. Fedorov, C. Mazelle, J.-J. Thocaven, M. Grande, Hannu E. J. Koskinen, T. Sales, W. Schmidt, P. Riihela, J. Kozyra, N. Krupp, J. Woch, M. Fränz, E. Dubinin, S. Orsini, R. Cerulli-Irelli, A. Mura, A. Milillo, M. Maggi, E. Roelof, P. Brandt, K. Szego, J. Scherrer, and P. Bochsler, Mars Express and Venus Express multi-point observations of geoeffective solar flare events in December 2006, Planet. Space Sci., 56, 873-880, 2008.

Häusler, B., M. Pätzold, G. L. Tyler, R. A. Simpson, M. K. Bird, V. Dehant, J.-P. Barriot, W. Eidel, R. Mattei, S. Remus, J. Selle, S. Tellmann, and T. Imamura, Radio science investigations by VeRa onboard the Venus Express spacecraft, Planet. Space Sci., 54, 1315-1335, 2006.

Häusler, B., M. Pätzold, G. L. Tyler, R. A. Simpson, D. Hinson, M. K. Bird, R. A. Treumann, V. Dehant, W. Eidel, S. Remus, and J. Selle, Atmospheric, ionospheric, surface, and radio wave propagation studies with the Venus Express radio science experiment VeRa, ESA Scientific Publication, ESA-SP-1295, 2009.

Hinson, D. P. and J. M. Jenkins, Magellan radio occultation measurements of atmospheric waves on Venus, Icarus, 114, 310-327, 1995.

Howard, H. T., G. L. Tyler, G. Fjeldbo, A. J. Kliore, G. S. Levy, D. L. Brunn, R. Dickinson, R. E. Edelson, W. L. Martin, R. B. Postal, B. Seidel, T. T. Sesplaukis, D. L. Shirley, C. T. Stelzried, D. N. Sweetnam, A. I. Zygielbaum, P. B. Esposito, J. D. Anderson, I. I. Shapiro, and R. D. Reasenberg, Venus: mass, gravity field, atmosphere, and ionosphere as measured by the Mariner 10 dual-frequency radio system, Science, 183, 1297-1301, 1974.

Imamura, T. and G. L. Hashimoto, Venus cloud formation in the meridional circulation, J. Geophys. Res., 103, 31349-31366, 1998.

Imamura, T. and G. L. Hashimoto, Microphysics of Venusian clouds in rising tropical air, J. Atmos. Sci., 58, 3597-3612, 2001.

Imamura, T., K. Noguchi, A. Nabatov, K.-I. Oyama, Z. Yamamoto, and M. Tokumaru, Phase scintillation observation during coronal sounding experiments with NOZOMI spacecraft, Astron. Astrophys., 439, 11651169, 2005.

Imamura, T., T. Iwata, Z. Yamamoto, N. Mochizuki, Y. Kono, K. Matsumoto, Q. Liu, H. Noda, H. Hanada, K.-I. Oyama, A. Nabatov, Y. Futaana, A. Saito, and H. Ando, Studying the lunar ionosphere with SELENE radio science experiment, Space Sci. Rev., 154, 305-316, 2010.

Jenkins, J. M. and P. G. Steffes, Results for 13-cm absorptivity and $\mathrm{H}_{2} \mathrm{SO}_{4}$ abundance profiles from the season 10 (1986) Pioneer Venus orbiter radio occultation experiment, Icarus, 90, 129-138, 1991.

Jenkins, J. M., P. G. Steffes, D. P. Hinson, J. D. Twicken, and G. L. Tyler, Radio occultation studies of the Venus atmosphere with the Magellan spacecraft. 2. Results from the October 1991 experiments, Icarus, 110, 79-93, 1994.

Karayel, E. T. and D. P. Hinson, Sub-Fresnel-scale vertical resolution in atmospheric profiles from radio occultation, Radio Sci., 32, 411-423, 1997.

Kliore, A. J., Radio occultation observations of the ionospheres of Mars and Venus, AGU Geophysical Monograph Series, 66, 265-276, 1992.

Kliore, A. J. and J. G. Luhmann, Solar cycle effects on the structure of the electron density profiles in the dayside ionosphere of Venus, J. Geophys. Res., 96, 21281-21289, 1991.

Kliore, A. J. and I. R. Patel, Vertical structure of the atmosphere of Venus from Pioneer Venus orbiter radio occultations, J. Geophys. Res., 85, 7957-7962, 1980.

Kliore, A. J. and I. R. Patel, Thermal structure of the atmosphere of Venus from Pioneer Venus radio occultations, Icarus, 52, 320-334, 1982.

Kliore, A. J., J. G. Luhmann, and M. H. G. Zhang, The effect of the solar cycle on the maintenance of the nightside ionosphere of Venus, J. Geophys. Res., 96, 11065-11071, 1991.

Leroy, S. S. and A. P. Ingersoll, Radio scintillations in Venus's atmosphere: application of a theory of gravity wave generation, J. Atmos. Sci., 53, 1018-1028, 1996.

Lipa, B. and L. Tyler, Statistical and computational uncertainties in atmospheric profiles from radio occultation: Mariner 10 at Venus, Icarus, 39, 192-208, 1979.

Markiewicz, W. J., D. V. Titov, S. S. Limaye, H. U. Keller, N. Ignatiev, R. Jaumann, N. Thomas, H. Michalik, R. Moissl, and P. Russo, Morphology and dynamics of the upper cloud layer of Venus, Nature, 450, 633-636, 2007.
Mizuno, E., N. Kawashima, T. Takano, and P. A. Rosen, Voyager radio science: observations and analysis of Neptune's atmosphere, IEICE Trans. Commun., E75-B, 665-672, 1992.

Nakamura, M., T. Imamura, M. Ueno, N. Iwagami, T. Satoh, S. Watanabe, M. Taguchi, Y. Takahashi, M. Suzuki, T. Abe, G. L. Hashimoto, T. Sakanoi, S. Okano, Y. Kasaba, J. Yoshida, M. Yamada, N. Ishii, T. Yamada, and K.-I. Oyama, PLANET-C: Venus Climate Orbiter mission of Japan, Planet. Space Sci., 55, 1831-1842, 2007.

Nakamura, M., T. Imamura, N. Ishii, T. Abe, T. Satoh, M. Suzuki, M. Ueno, A. Yamazaki, N. Iwagami, S. Watanabe, M. Taguchi, T. Fukuhara, Y. Takahashi, M. Yamada, N. Hoshino, S. Ohtsuki, K. Uemizu, G. L. Hashimoto, M. Takagi, Y. Matsuda, K. Ogohara, N. Sato, Y. Kasaba, T. Kouyama, N. Hirata, R. Nakamura, Y. Yamamoto, N. Okada, T. Horinouchi, M. Yamamoto, and Y. Hayashi, Overview of Venus orbiter, Akatsuki, Earth Planets Space, 63, 443-457, 2011.

Newman, M., G. Schubert, A. J. Kliore, and I. R. Patel, Zonal winds in the middle atmosphere of Venus from Pioneer Venus radio occultation data, J. Atmos. Sci., 41, 1901-1913, 1984.

Pätzold, M., J. Karl, and M. K. Bird, Coronal sounding with Ulysses: phase scintillation spectra in coronal holes and streamers, Astron. Astrophys., 316, 449-456, 1996.

Pätzold, M., B. Häusler, M. K. Bird, S. Tellmann, R. Mattei, S. W. Asmar, V. Dehant, W. Eidel, T. Imamura, R. A. Simpson, and G. L. Tyler, The structure of Venus' middle atmosphere and ionosphere, Nature, 450, 657-660, 2007a.

Pätzold, M. et al., Rosetta Radio Science Investigations (RSI), Space Sci. Rev., 128, 599-627, 2007b.

Rossow, W. B., A. D. Del Genio, S. S. Limaye, and L. D. Travis, Cloud morphology and motions from Pioneer Venus images, J. Geophys. Res., 85, 8107-8128, 1980.

Rossow, W. B., A. D. Del Genio, and T. Eichler, Cloud tracked winds from Pioneer Venus OCPP images, J. Atmos. Sci., 47, 2053-2084, 1990.

Seiff, A., J. T. Schofield, A. J. Kliore, F. W. Taylor, S. S. Limaye, H. E. Revercomb, L. A. Sromovsky, V. V. Kerzhanovich, V. I. Moroz, and M. Ya. Marov, Models of the structure of the atmosphere of Venus from the surface to 100 kilometers altitude, Adv. Space Res., 5(11), 3-58, 1985.

Takagi, M. and Y. Matsuda, Effects of thermal tides on the Venus atmospheric superrotation, J. Geophys. Res., 112, D09112, doi:10. 1029/2006JD007901, 2007.

Taylor, F. W., R. Beer, M. T. Chahine, D. J. Diner, L. S. Elson, R. D. Haskins, D. J. McCleese, J. V. Martonchik, P. E. Reichley, S. P. Bradley, J. Delderfield, J. T. Schofield, C. B. Farmer, L. Froidevaux, J. Leung, M. T. Coffey, and J. C. Gille, Structure and meteorology of the middle atmosphere of Venus: infrared remote sounding from the Pioneer Orbiter, J. Geophys. Res., 85, 7963-8006, 1980.

Tellmann, S., M. Pätzold, B. Häusler, M. K. Bird, and G. L. Tyler, Structure of the Venus neutral atmosphere as observed by the Radio Science experiment VeRa on Venus Express, J. Geophys. Res., 114, E00B36, doi:10.1029/2008JE003204, 2009.

Toigo, A., P. J. Gierasch, and M. D. Smith, High resolution cloud feature tracking on Venus by Galileo, Icarus, 109, 318-336, 1994.

Tyler, G. L., Radio propagation experiments in the outer solar system with Voyager, Proc. IEEE, 75, 1404-1431, 1987.

Woo, R. and J. W. Armstrong, Spacecraft radio scattering observations of the power spectrum of electron density fluctuations in the solar wind, $J$. Geophys. Res., 84, 7288-7296, 1979.

Woo, R., J. W. Armstrong, and A. Ishimaru, Radio occultation measurements of turbulence in the Venus atmosphere by Pioneer Venus, J. Geophys. Res., 85, 8031-8038, 1980.

Yamamoto, M. and H. Tanaka, Formation and maintenance of the 4-day circulation in the Venus middle atmosphere, J. Atmos. Sci., 54, 14721489, 1997.

Zhang, T. L., W. Baumjohann, M. Delva, H.-U. Auster, A. Balogh, C. T. Russell, S. Barabash, M. Balikhin, G. Berghofer, H. K. Biernat, H. Lammer, H. Lichtenegger, W. Magnes, R. Nakamura, T. Penz, K. Schwingenschuh, Z. Vörös, W. Zambelli, K.-H. Fornacon, K.-H. Glassmeier, I. Richter, C. Carr, K. Kudela, J. K. Shi, H. Zhao, U. Motschmann, and J.-P. Lebreton, Magnetic field investigation of the Venus plasma environment: Expected new results from Venus Express, Planet. Space Sci., 54, 1336-1343, 2006.

T. Imamura (e-mail: imamura.takeshi@jaxa.jp), T. Toda, A. Tomiki, D. Hirahara, T. Hayashiyama, N. Mochizuki, Z.-i. Yamamoto, T. Abe, T. Iwata, H. Noda, Y. Futaana, H. Ando, B. Häusler, M. Pätzold, and A. Nabatov 